\title{
Equivalence of Control Systems on the Pseudo-Orthogonal Group SO $(2,1)_{0}$
}

\author{
Rory Biggs and Claudiu C. Remsing
}

\begin{abstract}
We consider left-invariant control affine systems on the matrix Lie group SO $(2,1)_{0}$. A classification, under state space equivalence, of all such full-rank control systems is obtained. First, we identify certain subsets on which the group of Lie algebra automorphisms act transitively. We then systematically identify equivalence class representatives (for single-input, two-input and three-input control systems). A brief comparison of these classification results with existing results concludes the paper.
\end{abstract}

\section{Introduction}

From a geometric viewpoint, a (smooth) control system is given by a family of (smooth) vector fields parametrized by controls. An admissible trajectory of such a system, associated to a piecewise-constant control, is an integral curve of some vector field of the family or a finite concatenation of such curves. The arbitrary admissible control case can be realized via an approximation by piecewise-constant controls. Invariant control systems are control systems evolving on (real, finite dimensional) Lie groups with dynamics invariant under translations. Such systems were first considered in 1972 by Brockett [12] and by Jurdjevic and Sussmann [17]. For more details about (invariant) control systems see, e.g., [5], [16], [24], [6], [22].

Key Words: Left-invariant control system, state space equivalence, pseudo-orthogonal group.

2010 Mathematics Subject Classification: Primary 93A10, 93B17; Secondary 93B29, $22 \mathrm{E} 43$.

Received: 01.04.2015

Accepted: 30.06 .2015 
In order to understand the local geometry of control systems, one needs to introduce some natural equivalence relations. The most natural equivalence relation for control systems is equivalence up to coordinate changes in the state space. This is called state space equivalence (cf. [15], [10]). Two control systems are state space equivalent if they are related by a diffeomorphism (in which case their trajectories, corresponding to the same controls, are also related by that diffeomorphism). This equivalence relation is very strong. Consequently, there are so many equivalence classes that any general classification appears to be very difficult if not impossible. However, there is a chance for some reasonable classification in low dimensions. Another important equivalence relation for control systems is that of feedback equivalence (see, e.g., [23], [15]). Two feedback equivalent control systems have the same set of trajectories (up to a diffeomorphism in the state space) which are parametrized differently by admissible controls.

A systematic investigation of state space equivalence and feedback equivalence, in the context of left-invariant control systems, was recently carried out [10]. Incidentally, an appropriate specialization of feedback equivalence, called detached feedback equivalence, was also introduced. A classification, under state space equivalence, of invariant control systems evolving on the Euclidean group SE (2) was obtained in [2]. Classifications, under detached feedback equivalence, of various distinguished subclasses of invariant control systems have also been obtained in recent years (see, e.g., [7], [8], [9], [3], [1], $[4])$. Furthermore, an investigation of the equivalence of cost-extended control systems has been carried out in [11].

In this paper we consider only left-invariant control affine systems, evolving on a particular group, the pseudo-orthogonal group $\mathrm{SO}(2,1)_{0}$. We classify, under state space equivalence, all such full-rank control systems. Moreover, a representative for each equivalence class is identified in a systematic manner. A tabulation of these results is appended. Several problems related to control systems on SO $(2,1)_{0}$ (like controllability, stability, explicit integration by elliptic functions, numerical integration, and the existence of periodic solutions) have been considered in recent years (see [20], [19], [21], [13]).

\section{Invariant control systems and equivalence}

A left-invariant control affine system $\Sigma$ is a control system of the form

$$
\dot{g}=g \Xi(\mathbf{1}, u)=g\left(A+u_{1} B_{1}+\cdots+u_{\ell} B_{\ell}\right), \quad g \in \mathrm{G}, u \in \mathbb{R}^{\ell} .
$$

Here $G$ is a (real, finite-dimensional) matrix Lie group and the parametrization map $\Xi(\mathbf{1}, \cdot): \mathbb{R}^{\ell} \rightarrow \mathfrak{g}$ is an affine injection (i.e., $B_{1}, \ldots, B_{\ell}$ are linearly independent). The admissible controls are piecewise-continuous maps $u(\cdot)$ : 
$[0, T] \rightarrow \mathbb{R}^{\ell}$ and the trace of the system $\Gamma=A+\Gamma^{0}=A+\left\langle B_{1}, \ldots, B_{\ell}\right\rangle$ is an affine subspace of (the Lie algebra) $\mathfrak{g}$. A system $\Sigma$ is called homogeneous if $A \in \Gamma^{0}$, and inhomogeneous otherwise. Furthermore, $\Sigma$ has full rank provided the Lie algebra generated by its trace equals the whole Lie algebra $\mathfrak{g}$. Note that $\Sigma$ is completely determined by the specification of its state space $G$ and its parametrization map $\Xi(\mathbf{1}, \cdot)$. Hence, for a fixed $\mathrm{G}$, we shall specify $\Sigma$ by simply writing

$$
\Sigma: A+u_{1} B_{1}+\cdots+u_{\ell} B_{\ell} .
$$

If the state space $G$ of $\Sigma$ is a three-dimensional matrix Lie group, then the condition that $\Sigma$ has full rank can be characterized as follows. No homogeneous single-input system has full rank. An inhomogeneous single-input system has full rank if and only if $A, B_{1}$, and $\left[A, B_{1}\right]$ are linearly independent, whereas a homogeneous two-input system has full rank if and only if $B_{1}, B_{2}$, and $\left[B_{1}, B_{2}\right]$ are linearly independent. Also, it is clear that any inhomogeneous two-input or (homogeneous) three-input system has full rank. Henceforth we assume that all systems under consideration have full rank.

State space equivalence is well understood (cf. [5], [15]); it establishes a one-to-one correspondence between the trajectories of equivalent systems. Let $G$ be a fixed connected matrix Lie group and let $\Sigma$ and $\Sigma^{\prime}$ be two (leftinvariant control affine) systems on $\mathrm{G}$. We say that $\Sigma$ and $\Sigma^{\prime}$ are state space equivalent if there exist a diffeomorphism $\phi: \mathrm{G} \rightarrow \mathrm{G}$ such that $T_{g} \phi \cdot \Xi(g, u)=$ $\Xi^{\prime}(\phi(g), u)$ for all $g \in \mathrm{G}$ and $u \in \mathbb{R}^{\ell}$.

In this paper we shall refer to state space equivalence, simply, as equivalence. We recall an algebraic characterization of this equivalence.

Proposition 1 ([10]). Systems $\Sigma$ and $\Sigma^{\prime}$ are equivalent if and only if there exists a Lie algebra automorphism $\psi \in d$ Aut $(\mathrm{G})$ such that $\psi \cdot \Xi(\mathbf{1}, u)=$ $\Xi^{\prime}(\mathbf{1}, u)$ for all $u \in \mathbb{R}^{\ell}$.

Here $d$ Aut $(\mathrm{G})=\left\{T_{\mathbf{1}} \phi: \phi \in\right.$ Aut $\left.(\mathrm{G})\right\}$ is the subgroup of Lie algebra automorphisms, containing only linearized Lie group automorphisms.

It turns out that a classification of the $(\ell+1)$-input homogeneous systems may be (partially) obtained from a classification of the $\ell$-input inhomogeneous systems. Suppose $\left\{A^{i}+u_{1} B_{1}^{i}+\cdots+u_{\ell} B_{\ell}^{i}: i \in I\right\}$ is an exhaustive collection of equivalence class representatives for $\ell$-input inhomogeneous systems.

Lemma. If $\Sigma: A+u_{1} B_{1}+\cdots+u_{\ell+1} B_{\ell+1}$ is a $(\ell+1)$-input homogeneous system, then $\Sigma$ is equivalent to

$$
\widehat{\Sigma}_{i, \gamma}: \gamma_{1} B_{1}^{i}+\cdots+\gamma_{\ell} B_{\ell}^{i}+\gamma_{\ell+1} A^{i}+u_{1} B_{1}^{i}+\cdots+u_{\ell} B_{\ell}^{i}+u_{\ell+1} A^{i}
$$

for some $i \in I$ and some $\gamma_{1}, \ldots, \gamma_{\ell+1} \in \mathbb{R}$. 
Proof. $\Sigma^{\prime}: B_{\ell+1}+u_{1} B_{1}+\cdots+u_{\ell} B_{\ell}$ is a $\ell$-input inhomogeneous system. Thus (by proposition 1) there exists an automorphism $\psi \in d$ Aut (G) such $\psi \cdot B_{\ell+1}=A^{i}$ and $\psi \cdot B_{j}=B_{j}^{i}, 1 \leq j \leq \ell$ for some $i \in I$. Therefore $\Sigma$ is state space equivalent to $\Sigma^{\prime \prime}: \psi \cdot A+u_{1} B_{1}^{i}+\cdots+u_{\ell} B_{\ell}^{i}+u_{\ell+1} A^{i}$. However, as $\Sigma$ is homogeneous, so is $\Sigma^{\prime \prime}$. Hence $\psi \cdot A$ is a linear combination of $B_{1}^{i}, \ldots, B_{\ell}^{i}, A^{i}$, i.e., $\Sigma^{\prime \prime}=\widehat{\Sigma}_{i, \gamma}$.

Accordingly, $\left\{\widehat{\Sigma}_{i, \gamma}: i \in I, \gamma_{1}, \ldots, \gamma_{\ell+1} \in \mathbb{R}\right\}$ is an exhaustive collection of equivalence class representatives for $(\ell+1)$-input inhomogeneous systems. However, some of these systems may be equivalent to one another.

\section{The pseudo-orthogonal group $\mathrm{SO}(2,1)_{0}$}

The pseudo-orthogonal group

$$
\mathrm{SO}(2,1)=\left\{g \in \mathbb{R}^{3 \times 3}: g^{\top} J g=J, \operatorname{det} g=1\right\}
$$

is a three-dimensional simple Lie group. Here $J=\operatorname{diag}(1,1,-1)$. The identity component of $\mathrm{SO}(2,1)$ is $\mathrm{SO}(2,1)_{0}=\left\{g \in \mathrm{SO}(2,1): g_{33}>0\right\}$. Its Lie algebra

$$
\mathfrak{s o}(2,1)=\left\{A \in \mathbb{R}^{3 \times 3}: A^{\top} J+J A=0\right\}
$$

has an ordered basis

$$
E_{1}=\left[\begin{array}{lll}
0 & 0 & 0 \\
0 & 0 & 1 \\
0 & 1 & 0
\end{array}\right] \quad E_{2}=\left[\begin{array}{lll}
0 & 0 & 1 \\
0 & 0 & 0 \\
1 & 0 & 0
\end{array}\right] \quad E_{3}=\left[\begin{array}{ccc}
0 & 1 & 0 \\
-1 & 0 & 0 \\
0 & 0 & 0
\end{array}\right]
$$

The commutation operation is given by $\left[E_{2}, E_{3}\right]=E_{1},\left[E_{3}, E_{1}\right]=E_{2}$, and $\left[E_{1}, E_{2}\right]=-E_{3}$. The group Aut $(\mathfrak{s o}(2,1))$ of automorphisms of $\mathfrak{s o}(2,1)$ is exactly $\mathbf{S O}(2,1)$. Also, the group $\operatorname{Inn}(\mathfrak{s o}(2,1))$ of inner automorphisms of $\mathfrak{s o}(2,1)$ is exactly $\mathrm{SO}(2,1)_{0}$ (cf. [18]). (Here each automorphism $\psi$ is identified with its corresponding matrix $g$ with respect to the chosen basis.) We have that $\mathrm{SO}(2,1)$ is generated by

$$
\begin{aligned}
\rho_{2}(t) & =\left[\begin{array}{ccc}
\cosh t & 0 & \sinh t \\
0 & 1 & 0 \\
\sinh t & 0 & \cosh t
\end{array}\right] & \rho_{3}(t) & =\left[\begin{array}{ccc}
\cos t & \sin t & 0 \\
-\sin t & \cos t & 0 \\
0 & 0 & 1
\end{array}\right] \\
\eta(t) & =\left[\begin{array}{ccc}
1-\frac{1}{2} t^{2} & t & \frac{1}{2} t^{2} \\
-t & 1 & t \\
-\frac{1}{2} t^{2} & t & 1+\frac{1}{2} t^{2}
\end{array}\right] & \varsigma & =\left[\begin{array}{ccc}
-1 & 0 & 0 \\
0 & 1 & 0 \\
0 & 0 & -1
\end{array}\right] .
\end{aligned}
$$

Remark. $\rho_{2}(t)=\exp \left(t E_{2}\right), \rho_{3}(t)=\exp \left(t E_{3}\right)$, and $\eta(t)=\exp \left(t\left(E_{1}+E_{3}\right)\right)$. Also, $\rho_{2}(t), \rho_{3}(t), \eta(t) \in \operatorname{Inn}(\mathfrak{s o}(2,1))$, whereas $\varsigma \notin \operatorname{Inn}(\mathfrak{s o}(2,1))$. 
Proposition 2. The map $d$ : Aut $\left(\mathrm{SO}(2,1)_{0}\right) \rightarrow$ Aut $(\mathfrak{s o}(2,1)), \phi \mapsto T_{1} \phi$ is bijective.

Proof. As SO $(2,1)_{0}$ is connected, $d$ is injective (see, e.g., [14]). Furthermore, as $\rho_{2}(t), \rho_{3}(t), \eta(t) \in \operatorname{Inn}(\mathfrak{s o}(2,1))$ and the elements $\rho_{2}(t), \rho_{3}(t), \eta(t)$, and $\varsigma$ generate $\mathrm{SO}(2,1)=$ Aut $(\mathfrak{s o}(2,1))$, it suffices to show that $\varsigma \in d$ Aut $\left(\mathrm{SO}(2,1)_{0}\right)$. Let $\phi: \mathrm{SO}(2,1)_{0} \rightarrow \mathrm{SO}(2,1)_{0}, g \mapsto \varsigma g \varsigma$. We claim that $\phi$ is a Lie group automorphism such that $T_{\mathbf{1}} \phi=\varsigma$. Let $g \in \mathrm{SO}(2,1)_{0}$. Now $(\varsigma g \varsigma)^{\top} J(\varsigma g \varsigma)=\varsigma g^{\top} J g \varsigma=J$ and $\operatorname{det}(\varsigma g \varsigma)=\operatorname{det} \varsigma^{2} \operatorname{det} g=1$. Thus $\phi(g) \in \mathrm{SO}(2,1)$. Furthermore, the entry of the third column, third row of $g$ is fixed by $\phi$. Thus $\phi(g) \in \mathrm{SO}(2,1)_{0}$. As $\phi \circ \phi$ is the identity map on SO $(2,1)_{0}$, it follows that $\phi$ is bijective. Also, $\phi(g h)=\varsigma g h \varsigma=\varsigma g \varsigma \varsigma h \varsigma=$ $\phi(g) \phi(h)$. Finally, a simple calculation shows that $\phi\left(\exp \left(t E_{1}\right)\right)=\exp \left(\varsigma \cdot t E_{1}\right)$, $\phi\left(\exp \left(t E_{2}\right)\right)=\exp \left(\varsigma \cdot t E_{2}\right)$, and $\phi\left(\exp \left(t E_{3}\right)\right)=\exp \left(\varsigma \cdot t E_{3}\right)$. Thus $T_{\mathbf{1}} \phi=\varsigma$.

The (Lorentzian) product $\odot$ on $\mathfrak{s o}(2,1)$ is given by $A \odot B=a_{1} b_{1}+$ $a_{2} b_{2}-a_{3} b_{3}$. Here $A=\sum_{i=1}^{3} a_{i} E_{i}$ and $B=\sum_{i=1}^{3} b_{i} E_{i}$. Any automorphism $\psi$ preserves $\odot$, i.e., $(\psi \cdot A) \odot(\psi \cdot B)=A \odot B$. Consider the level sets $\mathcal{H}_{\alpha}=\{A \in \mathfrak{s o}(2,1): A \odot A=\alpha, A \neq 0\} . \mathcal{H}_{\alpha}$ is a hyperboloid of two sheets when $\alpha<0$, a hyperboloid of one sheet when $\alpha>0$, and a (punctured) cone when $\alpha=0$. As $\odot$ is preserved by automorphisms, each level set $\mathcal{H}_{\alpha}$ is also preserved. Moreover,

Proposition 3. The group Aut $(\mathfrak{s o}(2,1))$ acts transitively on each level set $\mathcal{H}_{\alpha}$.

Hence, for every $A \in \mathfrak{s o}(2,1)$, there exists $\psi \in \operatorname{Aut}(\mathfrak{s o}(2,1))$ such that $\psi \cdot A$ equals $\alpha E_{2}, \alpha E_{3}$, or $E_{1}+E_{3}$ for some $\alpha>0$. We now consider the subgroups of automorphisms fixing these respective vectors.

\section{Theorem 1.}

(i) The subgroup of Aut $(\mathfrak{s o}(2,1))$ fixing $E_{2}$ is $\left\{\rho_{2}(t), \varsigma \circ \rho_{2}(t): t \in \mathbb{R}\right\}$.

(ii) The subgroup of Aut $(\mathfrak{s o}(2,1))$ fixing $E_{3}$ is $\left\{\rho_{3}(t): t \in \mathbb{R}\right\}$.

(iii) The subgroup of Aut $(\mathfrak{s o}(2,1))$ fixing $E_{1}+E_{3}$ is $\{\eta(t): t \in \mathbb{R}\}$.

Proof. Let $\psi \in$ Aut $(\mathfrak{s o}(2,1))$ and let

$$
\psi=\left[\begin{array}{lll}
a_{1} & a_{2} & a_{3} \\
b_{1} & b_{2} & b_{3} \\
c_{1} & c_{2} & c_{3}
\end{array}\right] .
$$

Suppose $\psi \cdot E_{2}=E_{2}$. Then $a_{2}=c_{2}=0$ and $b_{2}=1$. The conditions $\psi^{\top} J \psi=J$ and $\operatorname{det} \psi=1$ then yield $b_{1}=b_{3}=0$ and $\left[\begin{array}{ll}a_{1} & a_{3} \\ c_{1} & c_{3}\end{array}\right] \in \mathrm{SO}(1,1)$. 
Therefore $\psi=\rho_{2}(t)$ or $\psi=\varsigma \circ \rho_{2}(t)$ for some $t \in \mathbb{R}$. Clearly $\left(\varsigma \circ \rho_{2}(t)\right) \cdot E_{2}=$ $E_{2}$ and $\rho_{2}(t) \cdot E_{2}=E_{2}$ for every $t \in \mathbb{R}$.

Suppose $\psi \cdot E_{3}=E_{3}$. Then $a_{3}=b_{3}=0$ and $c_{3}=1$. The conditions $\psi^{\top} J \psi=J$ and $\operatorname{det} \psi=1$ then yield $c_{1}=c_{2}=0$ and $\left[\begin{array}{ll}a_{1} & a_{2} \\ b_{1} & b_{2}\end{array}\right] \in \mathrm{SO}(2)$. Therefore $\psi=\rho_{3}(t)$ for some $t \in \mathbb{R}$. Clearly $\rho_{3}(t) \cdot E_{3}=E_{3}$ for every $t \in \mathbb{R}$.

Suppose $\psi \cdot\left(E_{1}+E_{3}\right)=E_{1}+E_{3}$. Then $a_{3}=1-a_{1}, b_{3}=-b_{1}$ and $c_{3}=1-c_{1}$. Again we impose the conditions $\psi^{\top} J \psi=J$ and $\operatorname{det} \psi=1$. A straightforward but tedious calculation then shows that $\psi=\eta(t)$ for some $t \in \mathbb{R}$. It is easy to verify that $\eta(t) \cdot\left(E_{1}+E_{3}\right)=E_{1}+E_{3}$.

Remark. The ordered basis for $\mathfrak{s o}(2,1)$ has been chosen so that Aut $(\mathfrak{s o}(2,1))=\mathrm{SO}(2,1)$. Indeed, with respect to this choice of basis, we have that the linear map ad $A=[A, \cdot]$ has matrix $\varsigma A \varsigma$. This accounts for the convenient situation that the subgroup of automorphisms fixing $E_{2}, E_{3}$, and $E_{1}+E_{3}$, respectively, are exactly $\exp \left(\mathbb{R} E_{2}\right) \cup\left(\varsigma \exp \left(\mathbb{R} E_{2}\right)\right), \exp \left(\mathbb{R} E_{3}\right)$, and $\exp \left(\mathbb{R}\left(E_{1}+E_{3}\right)\right)$, respectively.

Corollary 1. The only automorphism fixing at least two of $E_{1}, E_{2}, E_{3}$, and $E_{1}+E_{3}$ is the identity automorphism.

The subgroups of automorphisms fixing $E_{2}, E_{3}$, and $E_{1}+E_{3}$, respectively, preserve certain affine subspaces. Moreover, these subgroups are transitive on certain subsets of these affine subspaces. Let $A \in \mathfrak{s o}(2,1), A \neq 0, A=$ $a_{1} E_{1}+a_{2} E_{2}+a_{3} E_{3}$ and let

$$
\begin{aligned}
\Gamma_{2} & =a_{2} E_{2}+\left\langle E_{3}, E_{1}\right\rangle, & a_{1}^{2}-a_{3}^{2} \neq 0 \\
\Gamma_{2}^{\prime} & =\left(a_{2} E_{2}+\left\langle a_{1} E_{1}+a_{3} E_{3}\right\rangle\right) \backslash\left\{a_{2} E_{2}\right\}, & a_{1}^{2}-a_{3}^{2}=0 \\
\Gamma_{3} & =a_{3} E_{3}+\left\langle E_{1}, E_{2}\right\rangle, & a_{1}^{2}+a_{2}^{2} \neq 0 \\
\Gamma_{13} & =\left(a_{1}-a_{3}\right) E_{1}+\left\langle E_{2}, E_{1}+E_{3}\right\rangle, & a_{1} \neq a_{3} \\
\Gamma_{13}^{\prime} & =a_{2} E_{2}+\left\langle E_{1}+E_{3}\right\rangle, & a_{1}=a_{3} \text { and } a_{2} \neq 0 .
\end{aligned}
$$

(These sets are generated by considering the orthogonal compliments, with respect to $\odot$, of $\left\langle E_{2}\right\rangle,\left\langle E_{3}\right\rangle$, and $\left\langle E_{1}+E_{3}\right\rangle$.) If $A \notin\left\langle E_{2}\right\rangle$, then $A \in \Gamma_{2}$ or $A \in \Gamma_{2}^{\prime}$. If $A \notin\left\langle E_{3}\right\rangle$, then $A \in \Gamma_{3}$. If $A \notin\left\langle E_{1}+E_{3}\right\rangle$, then $A \in \Gamma_{13}$ or $A \in \Gamma_{13}^{\prime}$.

Proposition 4. Any automorphism $\rho_{2}(t)$ or $\varsigma \circ \rho_{2}(t)$ leaves $\Gamma_{2}$ and $\Gamma_{2}^{\prime}$ invariant. Any automorphism $\rho_{3}(t)$ leaves $\Gamma_{3}$ invariant. Any automorphism $\eta(t)$ leaves $\Gamma_{13}$ and $\Gamma_{13}^{\prime}$ invariant.

\section{Theorem 2.}


(i) The subgroup of Aut $(\mathfrak{s o}(2,1))$ fixing $E_{2}$ acts transitively on $\Gamma_{2} \cap \mathcal{H}_{A \odot A}$ and $\Gamma_{2}^{\prime} \cap \mathcal{H}_{A \odot A}$.

(ii) The subgroup of Aut $(\mathfrak{s o}(2,1))$ fixing $E_{3}$ acts transitively on $\Gamma_{3} \cap \mathcal{H}_{A \odot A}$.

(iii) The subgroup of Aut $(\mathfrak{s o}(2,1))$ fixing $E_{1}+E_{3}$ acts transitively on $\Gamma_{13} \cap$ $\mathcal{H}_{A \odot A}$ and $\Gamma_{13}^{\prime} \cap \mathcal{H}_{A \odot A}$.

We illustrate some of the typical cases in figures 1, 2, 3, and 4 .

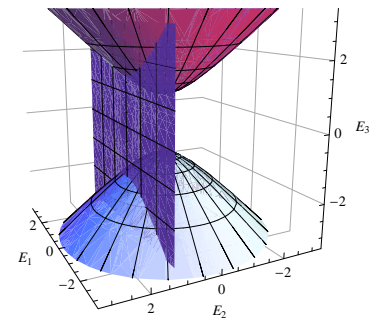

(a) $A \odot A<0$

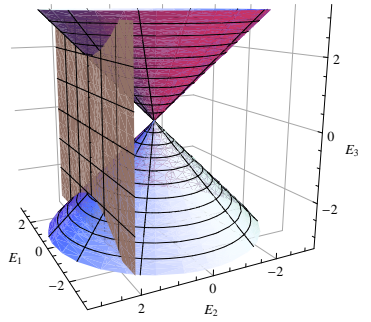

(b) $A \odot A=0$

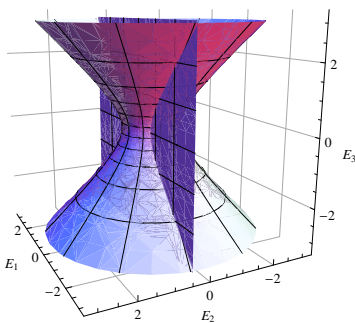

(c) $A \odot A>0$

Figure 1: Typical cases of $\Gamma_{2} \cap \mathcal{H}_{A \odot A}$

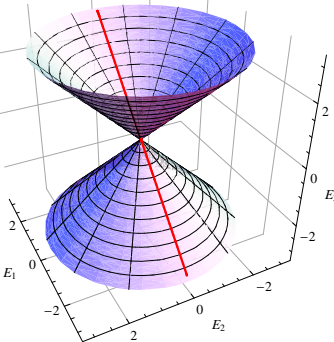

(a) $A \odot A=0$

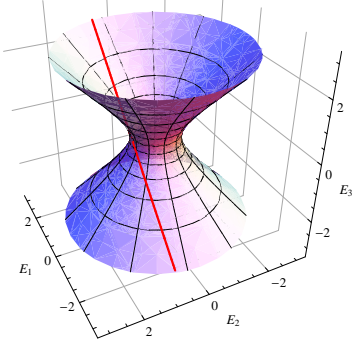

(b) $A \odot A>0$

Figure 2: Typical cases of $\Gamma_{2}^{\prime} \cap \mathcal{H}_{A \odot A}$ and $\Gamma_{13}^{\prime} \cap \mathcal{H}_{A \odot A}$

Proof. (i) By proposition 1, any automorphism $\psi$ fixing $E_{2}$ is of the form

$$
\psi=\left[\begin{array}{ccc}
k \cosh t & 0 & k \sinh t \\
0 & 1 & 0 \\
k \sinh t & 0 & k \cosh t
\end{array}\right]
$$

where $t \in \mathbb{R}$ and $k \in\{-1,1\}$. 


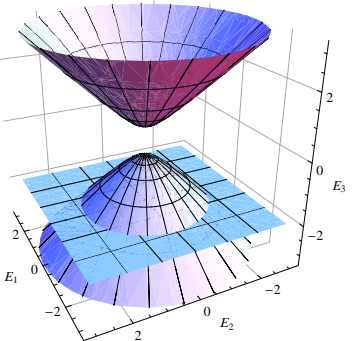

(a) $A \odot A<0$

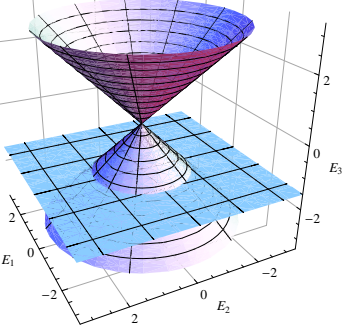

(b) $A \odot A=0$

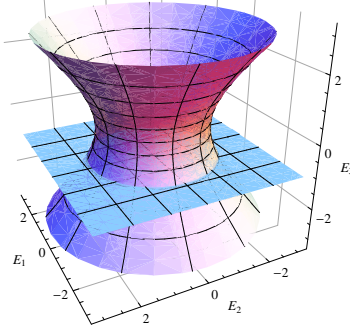

(c) $A \odot A>0$

Figure 3: Typical cases of $\Gamma_{3} \cap \mathcal{H}_{A \odot A}$

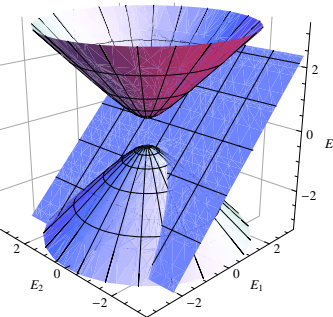

(a) $A \odot A<0$

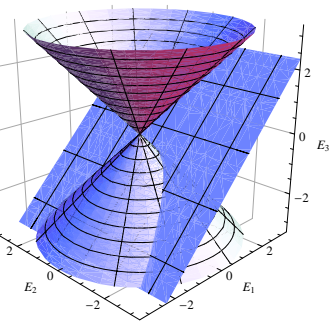

(b) $A \odot A=0$

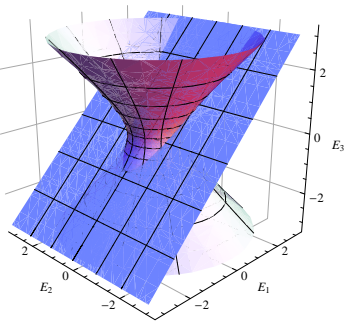

(c) $A \odot A>0$

Figure 4: Typical cases of $\Gamma_{13} \cap \mathcal{H}_{A \odot A}$ 
Suppose $a_{1}^{2}-a_{3}^{2} \neq 0$. Let $x E_{1}+y E_{2}+z E_{3} \in \Gamma_{2} \cap \mathcal{H}_{A \odot A}$. Then $y=a_{2}$ and $x^{2}-z^{2}=a_{1}^{2}-a_{3}^{2}$. It suffices to show that there exists and automorphism $\psi$ fixing $E_{2}$ such that $\psi \cdot A=x E_{1}+y E_{2}+z E_{3}$. Now $\psi \cdot A=k\left(a_{3} \sinh t+a_{1} \cosh t\right) E_{1}+a_{2} E_{2}+k\left(a_{1} \sinh t+a_{3} \cosh t\right) E_{3}$. Thus $\psi \cdot A=x E_{1}+y E_{2}+z E_{3}$ only if there exists $k \in\{-1,1\}$ and $t \in \mathbb{R}$ such that

$$
\left[\begin{array}{ll}
a_{1} & a_{3} \\
a_{3} & a_{1}
\end{array}\right]\left[\begin{array}{l}
k \cosh t \\
k \sinh t
\end{array}\right]=\left[\begin{array}{l}
x \\
z
\end{array}\right]
$$

Let $\left[\begin{array}{l}v_{1} \\ v_{2}\end{array}\right]=\left[\begin{array}{ll}a_{1} & a_{3} \\ a_{3} & a_{1}\end{array}\right]^{-1}\left[\begin{array}{l}x \\ z\end{array}\right]$ and let $k=\operatorname{sgn} v_{1}$. A simple calculation shows that $v_{1}^{2}-v_{2}^{2}=\frac{x^{2}-z^{2}}{a_{1}^{2}-a_{3}^{2}}=1$ (and so $v_{1} \neq 0$ ). There exists $t \in \mathbb{R}$ such that $k \sinh t=v_{2}$. Therefore $v_{1}^{2}=1-\sinh ^{2} t=\cosh ^{2} t$. Hence, as $k=\operatorname{sgn} v_{1}$, it follows that $v_{1}=k \cosh t$.

Suppose $a_{3}=a_{1} \neq 0$. Let $x E_{1}+y E_{2}+z E_{3} \in \Gamma_{2}^{\prime} \cap \mathcal{H}_{A \odot A}$. Then $y=a_{2}$ and $x=z \neq 0$. Now $\psi \cdot A=k e^{t} a_{1} E_{1}+a_{2} E_{2}+k e^{t} a_{1} E_{3}$. Hence there exists $k \in\{-1,1\}$ and $t \in \mathbb{R}$ such that $\psi \cdot A=x E_{1}+y E_{2}+z E_{3}$.

Suppose $a_{3}=-a_{1} \neq 0$. Let $x E_{1}+y E_{2}+z E_{3} \in \Gamma_{2}^{\prime} \cap \mathcal{H}_{A \odot A}$. Then $y=a_{2}$ and $x=-z \neq 0$. Now $\psi \cdot A=k e^{-t} a_{1} E_{1}+a_{2} E_{2}-k e^{-t} a_{1} E_{3}$. Hence there exists $k \in\{-1,1\}$ and $t \in \mathbb{R}$ such that $\psi \cdot A=x E_{1}+y E_{2}+z E_{3}$.

If $a_{1}=a_{3}=0$, then $\Gamma_{2}^{\prime}=\emptyset$.

(ii) By proposition 1, any automorphism $\psi$ fixing $E_{3}$ is of the form $\psi=$ $\rho_{3}(t)$ for some $t \in \mathbb{R}$. Let $x E_{1}+y E_{2}+z E_{3} \in \Gamma_{3} \cap \mathcal{H}_{A \odot A}$. Then $z=a_{3}$, $x^{2}+y^{2}=a_{1}^{2}+a_{2}^{2} \neq 0$. Now $\rho_{3}(t) \cdot A=\left(a_{2} \sin t+a_{1} \cos t\right) E_{1}+\left(a_{2} \cos t-\right.$ $\left.a_{1} \sin t\right) E_{2}+a_{3} E_{3}$. Thus $\rho_{3}(t) \cdot A=x E_{1}+y E_{2}+z E_{3}$ only if there exists $t \in \mathbb{R}$ such that

$$
\left[\begin{array}{cc}
a_{1} & a_{2} \\
a_{2} & -a_{1}
\end{array}\right]\left[\begin{array}{c}
\cos t \\
\sin t
\end{array}\right]=\left[\begin{array}{l}
x \\
y
\end{array}\right]
$$

Let $\left[\begin{array}{l}v_{1} \\ v_{2}\end{array}\right]=\left[\begin{array}{cc}a_{1} & a_{2} \\ a_{2} & -a_{1}\end{array}\right]^{-1}\left[\begin{array}{l}x \\ y\end{array}\right]$. Then $v_{1}^{2}+v_{2}^{2}=\frac{x^{2}+y^{2}}{a_{1}^{2}+a_{2}^{2}}=1$. Thus there does indeed exist a $t \in \mathbb{R}$ satisfying the above equation.

(iii) Again by proposition 1, any automorphism $\psi$ fixing $E_{1}+E_{3}$ is of the form $\psi=\eta(t)$ for some $t \in \mathbb{R}$. Now

$$
\begin{aligned}
\eta(t) \cdot A= & \left(a_{1}+a_{2} t+\frac{1}{2}\left(a_{3}-a_{1}\right) t^{2}\right) E_{1}+\left(a_{2}+\left(a_{3}-a_{1}\right) t\right) E_{2} \\
& +\left(a_{3}+a_{2} t+\frac{1}{2}\left(a_{3}-a_{1}\right) t^{2}\right) E_{3} .
\end{aligned}
$$

Suppose $a_{1} \neq a_{3}$ and let $x E_{1}+y E_{2}+z E_{3} \in \Gamma_{13} \cap \mathcal{H}_{A \odot A}$. Then $x=$ $a_{1}-a_{3}+z$ and $y^{2}=a_{1}^{2}+a_{2}^{2}-a_{3}^{2}+z^{2}-x^{2}$. Thus $\rho_{3}(t) \cdot A=x E_{1}+y E_{2}+z E_{3}$ 
only if there exists $t \in \mathbb{R}$ such that

$$
\left[\begin{array}{ccc}
a_{1} & a_{2} & \frac{1}{2}\left(a_{3}-a_{1}\right) \\
a_{2} & a_{3}-a_{1} & 0 \\
a_{3} & a_{2} & \frac{1}{2}\left(a_{3}-a_{1}\right)
\end{array}\right]\left[\begin{array}{c}
1 \\
t \\
t^{2}
\end{array}\right]=\left[\begin{array}{l}
x \\
y \\
z
\end{array}\right]
$$

The determinant of the above matrix equals $\frac{1}{2}\left(a_{1}-a_{3}\right)^{3}$ and so is nonzero. We have

$$
\begin{aligned}
{\left[\begin{array}{l}
v_{1} \\
v_{2} \\
v_{3}
\end{array}\right]=} & {\left[\begin{array}{ccc}
a_{1} & a_{2} & \frac{1}{2}\left(a_{3}-a_{1}\right) \\
a_{2} & a_{3}-a_{1} & 0 \\
a_{3} & a_{2} & \frac{1}{2}\left(a_{3}-a_{1}\right)
\end{array}\right]^{-1}\left[\begin{array}{c}
a_{1}-a_{3}+z \\
y \\
z
\end{array}\right] } \\
= & {\left[\begin{array}{c}
1 \\
\frac{a_{2}-y}{a_{1}-a_{3}} \\
\frac{2\left(a_{2}\left(-y+a_{2}\right)+\left(z-a_{3}\right)\left(-a_{1}+a_{3}\right)\right)}{\left(a_{1}-a_{3}\right)^{2}}
\end{array}\right] . }
\end{aligned}
$$

Let $t=v_{2}$. It is then a simple matter to verify (using the identity $y^{2}=$ $a_{1}^{2}+a_{2}^{2}-a_{3}^{2}+z^{2}-x^{2}$ ) that $v_{3}=t^{2}$. Therefore $\eta(t) \cdot A=x E_{1}+y E_{2}+z E_{3}$.

Suppose $a_{1}=a_{3}$ and $a_{2} \neq 0$. Let $x E_{1}+y E_{2}+z E_{3} \in \Gamma_{13}^{\prime} \cap \mathcal{H}_{A \odot A}$. Then $y=a_{2}$ and $x=z$. Now $\eta(t) \cdot A=\left(a_{1}+a_{2} t\right) E_{1}+a_{2} E_{2}+\left(a_{1}+a_{2} t\right) E_{3}$. So if $t=\frac{x-a_{1}}{a_{2}}$, then $\eta(t) \cdot A=x E_{1}+y E_{2}+z E_{3}$.

We shall find it useful to restate this result by identifying a typical point for each intersection. (This allows for easier application to classifying systems.)

\section{Corollary 2.}

1. Suppose $A \notin\left\langle E_{2}\right\rangle$.

(a) If $a_{1}^{2}-a_{3}^{2} \neq 0$, then there exists $t \in \mathbb{R}$ such that $\rho_{2}(t) \cdot A$ or $\left(\varsigma \circ \rho_{2}(t)\right) \cdot A$ equals $\left(\beta+\frac{1}{4}\right) E_{1}+a_{2} E_{2}+\left(\beta-\frac{1}{4}\right) E_{3}$, where $\beta=a_{1}^{2}-a_{3}^{2}$.

(b) If $a_{1}^{2}-a_{3}^{2}=0$, then there exists $t \in \mathbb{R}$ such that $\rho_{2}(t) \cdot A$ or $\left(\varsigma \circ \rho_{2}(t)\right) \cdot A$ equals $E_{1}+a_{2} E_{2}+k E_{3}$, where $k=\frac{a_{3}}{a_{1}}= \pm 1$.

2. Suppose $A \notin\left\langle E_{3}\right\rangle$. Then there exists $t \in \mathbb{R}$ such that $\rho_{3}(t) \cdot A=$ $\alpha E_{1}+a_{3} E_{3}$, where $\alpha=\sqrt{a_{1}^{2}+a_{2}^{2}}>0$.

3. Suppose $A \notin\left\langle E_{1}+E_{3}\right\rangle$.

(a) If $a_{1} \neq a_{3}$, then there exists $t \in \mathbb{R}$ such that $\eta(t) \cdot A=(\gamma+\beta) E_{1}+$ $\gamma E_{3}$, where $\gamma=a_{3}+\frac{a_{2}^{2}}{2 a_{1}-2 a_{3}}$ and $\beta=a_{1}-a_{3}$.

(b) If $a_{1}=a_{3}$, then there exists $t \in \mathbb{R}$ such that $\eta(t) \cdot A=E_{1}+\beta E_{2}+$ $E_{3}$, where $\beta=a_{2} \neq 0$. 


\section{Classification}

We now proceed to classify, under state space equivalence, all (full-rank) leftinvariant control affine systems on $\mathrm{SO}(2,1)_{0}$. This reduces (by propositions 1 and 2) to an algebraic classification of the corresponding affine parametrization maps. More precisely, $\Sigma$ and $\Sigma^{\prime}$ are equivalent if an only if there exists $\psi \in d$ Aut $\left(\mathrm{SO}(2,1)_{0}\right)=$ Aut $(\mathfrak{s o}(2,1))$ such that $\psi \cdot \Xi(\mathbf{1}, \cdot)=\Xi^{\prime}(\mathbf{1}, \cdot)$. We outline the approach to be used in classifying these systems. First, we distinguish between the number of controls involved and the homogeneity of the systems; this yields four types of systems. For each of these types, we simplify an arbitrary system by successively applying automorphisms. This simply involves applying proposition 3 and corollary 2 . Finally, we verify that all the candidates for class representatives are distinct and not equivalent. Families of these representatives are typically parametrized by some vectors $\boldsymbol{\alpha}=\left(\alpha_{i}\right)$, $\boldsymbol{\beta}=\left(\beta_{i}\right)$, and $\boldsymbol{\gamma}=\left(\gamma_{i}\right)$, where $\alpha_{i}>0, \beta_{i} \neq 0$, and $\gamma_{i} \in \mathbb{R}$.

When convenient, a system specified by

$$
\Sigma: \sum_{i=1}^{3} a_{i} E_{i}+u_{1} \sum_{i=1}^{3} b_{i} E_{i}+u_{2} \sum_{i=1}^{3} c_{i} E_{i}+u_{3} \sum_{i=1}^{3} d_{i} E_{i}
$$

will be represented as

$$
\left[\begin{array}{l|lll}
a_{1} & b_{1} & c_{1} & d_{1} \\
a_{2} & b_{2} & c_{2} & d_{2} \\
a_{3} & b_{3} & c_{3} & d_{3}
\end{array}\right] .
$$

The evaluation $\psi \cdot \Xi(\mathbf{1}, u)$ then becomes a matrix multiplication.

We start with single-input systems. (Only the inhomogeneous case need be considered as the homogeneous systems do not have full rank). The two-input homogeneous case follows as a corollary (by the lemma), although one still needs to verify that the systems obtained are not equivalent. (This verification shall be omitted as it is similar to the one made in the proof of the theorem.)

Theorem 3. Every single-input (inhomogeneous) system is equivalent to exactly one of the following systems

$$
\begin{aligned}
\Sigma_{1, \boldsymbol{\alpha} \gamma}^{(1,1)} & : \alpha_{2} E_{1}+\gamma_{1} E_{3}+u \alpha_{1} E_{3} \\
\Sigma_{2, \boldsymbol{\beta} \boldsymbol{\gamma}}^{(1,1)} & :\left(\gamma_{1}+\beta_{1}\right) E_{1}+\gamma_{1} E_{3}+u\left(E_{1}+E_{3}\right) \\
\Sigma_{3, \boldsymbol{\alpha} \boldsymbol{\beta} \boldsymbol{\gamma}}^{(1,1)} & :\left(\beta_{1}+\frac{1}{4}\right) E_{1}+\gamma_{1} E_{2}+\left(\beta_{1}-\frac{1}{4}\right) E_{3}+u \alpha_{1} E_{2} .
\end{aligned}
$$

Here $\alpha_{i}>0, \beta_{1} \neq 0$, and $\gamma_{1} \in \mathbb{R}$, with different values of these parameters yielding distinct (non-equivalent) class representatives. 
Proof. Let $\Sigma: A+u B$ be a single-input system.

Suppose $B \odot B<0$. Then (by proposition 3 ), there exists an automorphism $\psi$ such that $\psi \cdot B=\alpha_{1} E_{3}$ for some $\alpha_{1}>0$. Thus (by proposition 1) $\Sigma$ is equivalent to $\Sigma^{\prime}: A^{\prime}+u \alpha_{1} E_{3}$, where $A^{\prime}=\psi \cdot A$. Now, as $A$ and $B$ are linearly independent, $A^{\prime} \notin\left\langle E_{3}\right\rangle$. Hence (by corollary 2) there exists an automorphism $\psi^{\prime}$ such that $\psi^{\prime} \cdot \alpha_{1} E_{3}=\alpha_{1} E_{3}$ and $\psi^{\prime} \cdot A^{\prime}=\alpha_{2} E_{1}+\gamma_{1} E_{3}$ for some $\alpha_{2}>0$ and $\gamma_{1} \in \mathbb{R}$. Therefore $\Sigma^{\prime}$ (and so also $\Sigma$ ) is equivalent to $\Sigma_{1, \boldsymbol{\alpha} \boldsymbol{\gamma}}^{(1,1)}: \alpha_{2} E_{1}+\gamma_{1} E_{3}+u \alpha_{1} E_{3}$.

Suppose $B \odot B=0$. Then $\Sigma$ is equivalent to $\Sigma^{\prime}: A^{\prime}+u\left(E_{1}+E_{3}\right)$, where $A^{\prime} \notin\left\langle E_{1}+E_{3}\right\rangle$. Hence, $\Sigma$ is equivalent to either $\Sigma_{2, \boldsymbol{\beta} \gamma}^{(1,1)}:\left(\gamma_{1}+\beta_{1}\right) E_{1}+$ $\gamma_{1} E_{3}+u\left(E_{1}+E_{3}\right)$ or $\Sigma^{\prime \prime}: E_{1}+\beta_{1} E_{2}+E_{3}+u\left(E_{1}+E_{3}\right)$ for some $\gamma_{1} \in \mathbb{R}$ and $\beta_{1} \neq 0$. However, $\Sigma^{\prime \prime}$ does not have full rank. As the full rank property is preserved by equivalence, it follows that $\Sigma$ is equivalent to $\Sigma_{2, \boldsymbol{\beta} \gamma}^{(1,1)}$.

Suppose $B \odot B>0$. Then $\Sigma$ is equivalent to $\Sigma^{\prime}: A^{\prime}+u \alpha_{1} E_{2}$ for some $\alpha_{1}>0$, where $A^{\prime} \notin\left\langle E_{2}\right\rangle$. Hence, $\Sigma$ is equivalent to either $\Sigma_{3, \boldsymbol{\alpha} \boldsymbol{\beta} \boldsymbol{\gamma}}^{(1,1)}$ : $\left(\beta_{1}+\frac{1}{4}\right) E_{1}+\gamma_{1} E_{2}+\left(\beta_{1}-\frac{1}{4}\right) E_{3}+u \alpha_{1} E_{2}$ or $\Sigma^{\prime \prime}: E_{1}+\gamma_{1} E_{2}+E_{3}+u \alpha_{1} E_{2}$ for some $\gamma_{1} \in \mathbb{R}$ and $\beta_{1} \neq 0$. However, $\Sigma^{\prime \prime}$ does not have full rank and so $\Sigma$ is equivalent to $\Sigma_{3, \boldsymbol{\alpha} \boldsymbol{\beta} \boldsymbol{\gamma}}^{(1,1)}$.

It remains to be shown that no two of these equivalence representatives are equivalent. Let $\Sigma: A+u B$. If $\Sigma=\Sigma_{1, \boldsymbol{\alpha} \boldsymbol{\gamma}}^{(1,1)}$, then $B \odot B<0$. If $\Sigma=\Sigma_{2, \boldsymbol{\beta} \boldsymbol{\gamma}}^{(1,1)}$, then $B \odot B=0$. If $\Sigma=\Sigma_{3, \boldsymbol{\alpha} \boldsymbol{\beta} \boldsymbol{\gamma}}^{(1,1)}$, then $B \odot B>0$. Thus, as $\odot$ is preserved by any automorphism, $\Sigma_{1, \boldsymbol{\alpha} \boldsymbol{\gamma}}^{(1,1)}$ is not equivalent to either $\Sigma_{2, \boldsymbol{\beta} \boldsymbol{\gamma}}^{(1,1)}$ or $\Sigma_{3, \boldsymbol{\alpha} \boldsymbol{\beta} \boldsymbol{\gamma}}^{(1,1)}$ Likewise $\Sigma_{2, \boldsymbol{\beta} \gamma}^{(1,1)}$ is not equivalent to $\Sigma_{3, \boldsymbol{\alpha} \boldsymbol{\beta} \boldsymbol{\gamma}}^{(1,1)}$.

Suppose $\Sigma_{1, \boldsymbol{\alpha} \boldsymbol{\gamma}}^{(1,1)}$ is equivalent to $\Sigma_{1, \boldsymbol{\alpha}^{\prime} \boldsymbol{\gamma}^{\prime}}^{(1,1}$. Then there exists an automorphism $\psi$ such that

$$
\psi \cdot\left[\begin{array}{c|c}
\alpha_{2} & 0 \\
0 & 0 \\
\gamma_{1} & \alpha_{1}
\end{array}\right]=\left[\begin{array}{c|c}
\alpha_{2}^{\prime} & 0 \\
0 & 0 \\
\gamma_{1}^{\prime} & \alpha_{1}^{\prime}
\end{array}\right] .
$$

Thus $-\alpha_{1}^{2}=\alpha_{1} E_{3} \odot \alpha_{1} E_{3}=\alpha_{1}^{\prime} E_{3} \odot \alpha_{1}^{\prime} E_{3}=-\alpha_{1}^{\prime 2}$. Hence, as $\alpha_{1}, \alpha_{1}^{\prime}>0$, $\alpha=\alpha^{\prime}$. Thus $\psi \cdot E_{3}=E_{3}$. Therefore (by proposition 1 ) $\psi=\rho_{3}(t)$ for some $t>0$. Then it follows that $\gamma_{1}=\gamma_{1}^{\prime}$ and $\alpha_{2}=\alpha_{2}^{\prime}$. That is to say $\Sigma_{1, \boldsymbol{\alpha} \gamma}^{(1,1)}$ and $\Sigma_{1, \boldsymbol{\alpha}^{\prime} \boldsymbol{\gamma}^{\prime}}^{(1,1)}$ are equivalent only if $\boldsymbol{\alpha}=\boldsymbol{\alpha}^{\prime}$ and $\gamma_{1}=\gamma_{1}^{\prime}$.

Suppose $\Sigma_{2, \boldsymbol{\beta} \boldsymbol{\gamma}}^{(1,1)}$ is equivalent to $\Sigma_{2, \boldsymbol{\beta}^{\prime} \boldsymbol{\gamma}^{\prime}}^{(1,1)}$. Then there exists an automorphism $\psi$ such that

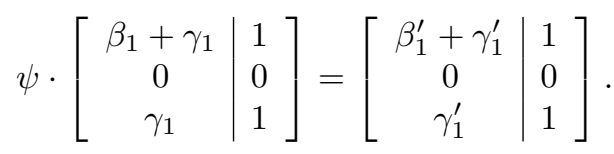


Hence, as $\psi \cdot\left(E_{1}+E_{3}\right)=E_{1}+E_{3}, \psi=\eta(t)$ for some $t \in \mathbb{R}$. We have

$$
\eta(t) \cdot\left[\begin{array}{c|c}
\beta_{1}+\gamma_{1} & 1 \\
0 & 0 \\
\gamma_{1} & 1
\end{array}\right]=\left[\begin{array}{c|c}
\beta_{1}-\frac{t^{2} \beta_{1}}{2}+\gamma_{1} & 1 \\
-t \beta_{1} & 0 \\
-\frac{t^{2} \beta_{1}}{2}+\gamma_{1} & 1
\end{array}\right] .
$$

Therefore $t=0$ and so $\psi$ is the identity automorphism. Consequently $\Sigma_{2, \boldsymbol{\beta} \boldsymbol{\gamma}}^{(1,1)}$ and $\Sigma_{2, \boldsymbol{\beta}^{\prime} \boldsymbol{\gamma}^{\prime}}^{(1,1)}$ are equivalent only if $\beta_{1}=\beta_{1}^{\prime}$ and $\gamma_{1}=\gamma_{1}^{\prime}$.

Similar computations show that $\Sigma_{3, \boldsymbol{\alpha} \boldsymbol{\beta} \boldsymbol{\gamma}}^{(1,1)}$ is equivalent to $\Sigma_{3, \boldsymbol{\alpha}^{\prime} \boldsymbol{\beta}^{\prime} \boldsymbol{\gamma}^{\prime}}^{(1,1)}$ only if $\boldsymbol{\alpha}=\boldsymbol{\alpha}^{\prime}, \boldsymbol{\beta}=\boldsymbol{\beta}^{\prime}$ and $\boldsymbol{\gamma}=\boldsymbol{\gamma}^{\prime}$.

Corollary 3. Every two-input homogeneous system is equivalent to exactly one of the following systems

$$
\begin{aligned}
& \Sigma_{1, \boldsymbol{\alpha} \boldsymbol{\gamma}}^{(2,0)}: \gamma_{3} E_{1}+\gamma_{2} E_{3}+u_{1}\left(\alpha_{2} E_{1}+\gamma_{1} E_{3}\right)+u_{2} \alpha_{1} E_{3} \\
& \Sigma_{2, \boldsymbol{\beta} \boldsymbol{\gamma}}^{(2,0)}: \gamma_{3} E_{1}+\gamma_{2} E_{3}+u_{1}\left(\left(\gamma_{1}+\beta_{1}\right) E_{1}+\gamma_{1} E_{3}\right)+u_{2}\left(E_{1}+E_{3}\right) \\
& \Sigma_{3, \boldsymbol{\alpha} \boldsymbol{\beta} \boldsymbol{\gamma}}^{(2,0)}: \gamma_{2}\left(\beta_{1}+\frac{1}{4}\right) E_{1}+\gamma_{3} E_{2}+\gamma_{2}\left(\beta_{1}-\frac{1}{4}\right) E_{3} \\
& \quad+u_{1}\left(\left(\beta_{1}+\frac{1}{4}\right) E_{1}+\gamma_{1} E_{2}+\left(\beta_{1}-\frac{1}{4}\right) E_{3}\right)+u_{2} \alpha_{1} E_{2} .
\end{aligned}
$$

Here $\alpha_{i}>0, \beta_{1} \neq 0$, and $\gamma_{i} \in \mathbb{R}$, with different values of these parameters yielding distinct (non-equivalent) class representatives.

Next we deal with the two-input inhomogeneous systems. The three-input case then follows as a corollary (as all three-input systems are clearly homogeneous).

Theorem 4. Every two-input inhomogeneous system is equivalent to exactly one of the following systems

$$
\begin{aligned}
& \Sigma_{1, \boldsymbol{\alpha} \boldsymbol{\beta} \boldsymbol{\gamma}}^{(2,1)}: \gamma_{3} E_{1}+\beta_{1} E_{2}+\gamma_{2} E_{3}+u_{1}\left(\alpha_{2} E_{1}+\gamma_{1} E_{3}\right)+u_{2} \alpha_{1} E_{3} \\
& \Sigma_{2, \boldsymbol{\beta} \gamma}^{(2,1)}: \gamma_{3} E_{1}+\beta_{2} E_{2}+\gamma_{2} E_{3}+u_{1}\left(\left(\gamma_{1}+\beta_{1}\right) E_{1}+\gamma_{1} E_{3}\right)+u_{2}\left(E_{1}+E_{3}\right) \\
& \Sigma_{3, \boldsymbol{\beta} \gamma}^{(2,1)}: \gamma_{1} E_{1}+\gamma_{2} E_{2}+\left(\beta_{2}+\gamma_{1}\right) E_{3}+u_{1}\left(E_{1}+\beta_{1} E_{2}+E_{3}\right)+u_{2}\left(E_{1}+E_{3}\right) \\
& \Sigma_{4, \boldsymbol{\alpha} \boldsymbol{\beta} \boldsymbol{\gamma}}^{(2,1)}:\left(\beta_{2}\left(\beta_{1}-\frac{1}{4}\right)+\gamma_{2}\left(\beta_{1}+\frac{1}{4}\right)\right) E_{1}+\left(\beta_{2}\left(\beta_{1}+\frac{1}{4}\right)+\gamma_{2}\left(\beta_{1}-\frac{1}{4}\right)\right) E_{3} \\
& \quad+\gamma_{3} E_{2}+u_{1}\left(\left(\beta_{1}+\frac{1}{4}\right) E_{1}+\gamma_{1} E_{2}+\left(\beta_{1}-\frac{1}{4}\right) E_{3}\right)+u_{2} \alpha_{1} E_{2} \\
& \Sigma_{5, \boldsymbol{\alpha} \boldsymbol{\beta} \boldsymbol{\gamma}}^{(2,1)}: \gamma_{3} E_{1}+\gamma_{2} E_{2}+\left(\beta_{1}+\gamma_{3}\right) E_{3}+u_{1}\left(E_{1}+\gamma_{1} E_{2}+E_{3}\right)+u_{2} \alpha_{1} E_{2} .
\end{aligned}
$$

Here $\alpha_{i}>0, \beta_{i} \neq 0$, and $\gamma_{i} \in \mathbb{R}$, with different values of these parameters yielding distinct (non-equivalent) class representatives. 
Proof. Let $\Sigma: A+u_{1} B_{1}+u_{2} B_{2}$ be a two-input system.

Suppose $B_{2} \odot B_{2}<0$. Then $\Sigma$ is equivalent to $\Sigma^{\prime}: A^{\prime}+u_{1} B_{1}^{\prime}+\alpha_{1} E_{3}$ for some $\alpha_{1}>0$, where $B_{1}^{\prime} \notin\left\langle E_{3}\right\rangle$. Hence $\Sigma$ is equivalent to $\Sigma_{1, \boldsymbol{\alpha} \boldsymbol{\beta} \boldsymbol{\gamma}}^{(2,1)}$ : $\gamma_{3} E_{1}+\beta_{1} E_{2}+\gamma_{2} E_{3}+u_{1}\left(\alpha_{2} E_{1}+\gamma_{1} E_{3}\right)+u_{2} \alpha_{1} E_{3}$ for some $\alpha_{2}>0$ and $\gamma_{1}, \gamma_{2}, \gamma_{3}, \beta_{1} \in \mathbb{R}$. As $\Sigma_{1, \boldsymbol{\alpha} \boldsymbol{\beta} \boldsymbol{\gamma}}^{(2,1)}$ is inhomogeneous, it follows that $\beta_{1} \neq 0$.

Suppose $B_{2} \odot B_{2}=0$. Then $\Sigma$ is equivalent to $\Sigma^{\prime}: A^{\prime}+u_{1} B_{1}^{\prime}+u_{2}\left(E_{1}+\right.$ $\left.E_{3}\right)$, where $B^{\prime} \notin\left\langle E_{1}+E_{3}\right\rangle$. Hence, $\Sigma$ is equivalent to either $\Sigma_{2, \boldsymbol{\beta} \boldsymbol{\gamma}}^{(2,1)}: \gamma_{3} E_{1}+$ $\beta_{2} E_{2}+\gamma_{2} E_{3}+u_{1}\left(\left(\gamma_{1}+\beta_{1}\right) E_{1}+\gamma_{1} E_{3}\right)+u_{2}\left(E_{1}+E_{3}\right)$ or $\Sigma_{3, \boldsymbol{\beta} \gamma}^{(2,1)}: \gamma_{1} E_{1}+\gamma_{2} E_{2}+$ $\left(\beta_{2}+\gamma_{1}\right) E_{3}+u_{1}\left(E_{1}+\beta_{1} E_{2}+E_{3}\right)+u_{2}\left(E_{1}+E_{3}\right)$ for some $\gamma_{1}, \gamma_{2}, \gamma_{3}, \beta_{2} \in \mathbb{R}$ and $\beta_{1} \neq 0$. As $\Sigma_{2, \boldsymbol{\beta} \boldsymbol{\gamma}}^{(2,1)}$ and $\Sigma_{3, \boldsymbol{\beta} \boldsymbol{\gamma}}^{(2,1)}$ are inhomogeneous, it follows that $\beta_{2} \neq 0$.

Suppose $B_{2} \odot B_{2}>0$. Then $\Sigma$ is equivalent to $\widetilde{\Sigma}: \tilde{A}+u_{1} \tilde{B}_{1}+u_{2} \alpha_{1} E_{2}$ for some $\alpha_{1}>0$, where $\tilde{B}_{1} \notin\left\langle E_{2}\right\rangle$. Hence, $\Sigma$ is equivalent to either $\Sigma^{\prime}$ : $A^{\prime}+u_{1}\left(\left(\beta_{1}+\frac{1}{4}\right) E_{1}+\gamma_{1} E_{2}+\left(\beta_{1}-\frac{1}{4}\right) E_{3}\right)+u_{2} \alpha_{1} E_{2}$ or $\Sigma^{\prime \prime}: A^{\prime \prime}+u_{1}\left(E_{1}+\right.$ $\left.\gamma_{1} E_{2}+E_{3}\right)+u_{2} \alpha_{1} E_{2}$ for some $\gamma_{1} \in \mathbb{R}$ and $\beta_{1} \neq 0$. We require that $A^{\prime}$, $\left(\beta_{1}+\frac{1}{4}\right) E_{1}+\gamma_{1} E_{2}+\left(\beta_{1}-\frac{1}{4}\right) E_{3}$, and $\alpha_{1} E_{2}$ are linearly independent. We have that $\left(\beta_{1}-\frac{1}{4}\right) E_{1}+\left(\beta_{1}+\frac{1}{4}\right) E_{3},\left(\beta_{1}+\frac{1}{4}\right) E_{1}+\left(\beta_{1}-\frac{1}{4}\right) E_{3}$, and $\alpha_{3} E_{3}$ are linearly independent. Thus $A^{\prime}=\left(\beta_{2}\left(\beta_{1}-\frac{1}{4}\right)+\gamma_{2}\left(\beta_{1}+\frac{1}{4}\right)\right) E_{1}+\gamma_{3} E_{2}+$ $\left(\beta_{2}\left(\beta_{1}+\frac{1}{4}\right)+\gamma_{2}\left(\beta_{1}-\frac{1}{4}\right)\right) E_{3}$ for some $\gamma_{2}, \gamma_{3} \in \mathbb{R}$ and $\beta_{2} \neq 0$. Hence $\Sigma^{\prime}=$ $\Sigma_{4, \boldsymbol{\alpha} \boldsymbol{\beta} \gamma}^{(2,1)}$. We also require that $A^{\prime \prime}, E_{1}+\gamma_{1} E_{2}+E_{3}$, and $\alpha_{1} E_{2}$ are linearly independent. Thus $A^{\prime \prime}=\gamma_{3} E_{1}+\gamma_{2} E_{2}+\left(\beta_{1}+\gamma_{3}\right) E_{3}$ for some $\gamma_{2}, \gamma_{3} \in \mathbb{R}$ and $\beta_{1} \neq 0$. Therefore $\Sigma^{\prime \prime}=\Sigma_{5, \boldsymbol{\alpha} \boldsymbol{\beta} \boldsymbol{\gamma}}^{(2,1)}$.

It remains to be shown that no two of these equivalence representatives are equivalent. As $\odot$ is preserved by any automorphism, it follows that $\Sigma_{1, \boldsymbol{\alpha} \boldsymbol{\beta} \boldsymbol{\gamma}}^{(2,1)}$ is not equivalent to $\Sigma_{2, \boldsymbol{\beta} \boldsymbol{\gamma}}^{(2,1)}, \Sigma_{3, \boldsymbol{\beta} \boldsymbol{\gamma}}^{(2,1)}, \Sigma_{4, \boldsymbol{\alpha} \boldsymbol{\beta} \boldsymbol{\gamma}}^{(2,1)}$, or $\Sigma_{5, \boldsymbol{\alpha} \boldsymbol{\beta} \boldsymbol{\gamma}}^{(2,1)}$. Likewise, $\Sigma_{2, \boldsymbol{\beta} \boldsymbol{\gamma}}^{(2,1)}$ is not equivalent to $\Sigma_{4, \boldsymbol{\alpha} \boldsymbol{\beta} \boldsymbol{\gamma}}^{(2,1)}$ or $\Sigma_{5, \boldsymbol{\alpha} \boldsymbol{\beta} \boldsymbol{\gamma}}^{(2,1)} ; \Sigma_{3, \boldsymbol{\beta} \boldsymbol{\gamma}}^{(2,1)}$ is not equivalent to $\Sigma_{4, \boldsymbol{\alpha} \boldsymbol{\beta} \boldsymbol{\gamma}}^{(2,1)}$ or $\Sigma_{5, \boldsymbol{\alpha} \boldsymbol{\beta} \boldsymbol{\gamma}}^{(2,1)}$

Suppose $\Sigma_{3, \boldsymbol{\beta} \boldsymbol{\gamma}}^{(2,1)}$ is equivalent to $\Sigma_{2, \boldsymbol{\beta}^{\prime} \boldsymbol{\gamma}^{\prime}}^{(2,1)}$. Then there exists an automorphism $\eta(t)$ fixing $E_{1}+E_{3}$ such that

$$
\eta(t) \cdot\left[\begin{array}{c|cc}
\gamma_{1} & 1 & 1 \\
\gamma_{2} & \beta_{1} & 0 \\
\beta_{2}+\gamma_{1} & 1 & 1
\end{array}\right]=\left[\begin{array}{c|cc}
\gamma_{3} & \beta_{1}+\gamma_{1} & 1 \\
\beta_{2} & 0 & 0 \\
\gamma_{2} & \gamma_{1} & 1
\end{array}\right]
$$

i.e.,

$$
\left[\begin{array}{c|cc}
\frac{t^{2} \beta_{2}}{2}+\gamma_{1}+t \gamma_{2} & 1+t \beta_{1} & 1 \\
t \beta_{2}+\gamma_{2} & \beta_{1} & 0 \\
\frac{1}{2}\left(2+t^{2}\right) \beta_{2}+\gamma_{1}+t \gamma_{2} & 1+t \beta_{1} & 1
\end{array}\right]=\left[\begin{array}{c|cc}
\gamma_{3} & \beta_{1}+\gamma_{1} & 1 \\
\beta_{2} & 0 & 0 \\
\gamma_{2} & \gamma_{1} & 1
\end{array}\right]
$$


Thus $\beta_{1}=0$, a contradiction. Hence $\Sigma_{3, \boldsymbol{\beta} \boldsymbol{\gamma}}^{(2,1)}$ is not equivalent to $\Sigma_{2, \boldsymbol{\beta}^{\prime} \boldsymbol{\gamma}^{\prime}}^{(2,1)}$ (for any admissible parameters). Similarly, $\Sigma_{4, \boldsymbol{\alpha} \boldsymbol{\beta} \boldsymbol{\gamma}}^{(2,1)}$ is not equivalent to $\Sigma_{5, \boldsymbol{\alpha}^{\prime} \boldsymbol{\beta}^{\prime} \boldsymbol{\gamma}^{\prime}}^{(2,1)}$.

Suppose $\Sigma_{1, \boldsymbol{\alpha} \boldsymbol{\beta} \boldsymbol{\gamma}}^{(2,1)}$ is equivalent to $\Sigma_{1, \boldsymbol{\alpha}^{\prime} \boldsymbol{\beta}^{\prime} \boldsymbol{\gamma}^{\prime}}^{(2,1)}$. Then there exists an automorphism $\psi$ such that

$$
\psi \cdot\left[\begin{array}{c|cc}
\gamma_{3} & \alpha_{2} & 0 \\
\beta_{1} & 0 & 0 \\
\gamma_{2} & \gamma_{1} & \alpha_{1}
\end{array}\right]=\left[\begin{array}{c|cc}
\gamma_{3}^{\prime} & \alpha_{2}^{\prime} & 0 \\
\beta_{1}^{\prime} & 0 & 0 \\
\gamma_{2}^{\prime} & \gamma_{1}^{\prime} & \alpha_{1}^{\prime}
\end{array}\right]
$$

Thus $-\alpha_{1}^{2}=-\alpha_{1}^{\prime 2}$ and so $\alpha_{1}=\alpha_{1}^{\prime}$. Therefore $\psi$ fixes $E_{3}$. Hence $\psi=\rho_{3}(t)$ for some $t \in \mathbb{R}$. Now

$$
\rho_{3}(t) \cdot\left[\begin{array}{c|cc}
\gamma_{3} & \alpha_{2} & 0 \\
\beta_{1} & 0 & 0 \\
\gamma_{2} & \gamma_{1} & \alpha_{1}
\end{array}\right]=\left[\begin{array}{c|cc}
\beta_{1} \sin t+\gamma_{3} \cos t & \alpha_{2} \cos t & 0 \\
\beta_{1} \cos t-\gamma_{3} \sin t & -\alpha_{2} \sin t & 0 \\
\gamma_{2} & \gamma_{1} & \alpha_{1}
\end{array}\right] .
$$

Thus $\gamma_{1}=\gamma_{1}^{\prime}$. Therefore $\alpha_{2}^{2}=\alpha_{2}^{\prime 2}$ and so $\alpha_{2}=\alpha_{2}^{\prime}$. Hence $\psi \cdot \alpha_{2} E_{1}=\alpha_{2} E_{1}$, i.e., $\psi$ fixes $E_{1}$. Hence, (by corollary 1 ) $\psi$ is the identity automorphism. Accordingly $\Sigma_{1, \boldsymbol{\alpha} \boldsymbol{\beta} \boldsymbol{\gamma}}^{(2,1)}$ is equivalent to $\Sigma_{1, \boldsymbol{\alpha}^{\prime} \boldsymbol{\beta}^{\prime} \boldsymbol{\gamma}^{\prime}}^{(2,1)}$ only if $\boldsymbol{\alpha}=\boldsymbol{\alpha}^{\prime}, \beta_{1}=\beta_{1}^{\prime}$, and $\gamma=\gamma^{\prime}$

Suppose $\Sigma_{4, \boldsymbol{\alpha} \boldsymbol{\beta} \boldsymbol{\gamma}}^{(2,1)}$ is equivalent to $\Sigma_{4, \boldsymbol{\alpha}^{\prime} \boldsymbol{\beta}^{\prime} \boldsymbol{\gamma}^{\prime}}^{(2,1)}$. Then there exists an automorphism $\psi$ such that

$$
\begin{array}{r}
\psi \cdot\left[\begin{array}{c|cc}
\left(\beta_{1}-\frac{1}{4}\right) \beta_{2}+\left(\beta_{1}+\frac{1}{4}\right) \gamma_{2} & \beta_{1}+\frac{1}{4} & 0 \\
\gamma_{3} & \gamma_{1} & \alpha_{1} \\
\left(\beta_{1}+\frac{1}{4}\right) \beta_{2}+\left(\beta_{1}-\frac{1}{4}\right) \gamma_{2} & \beta_{1}-\frac{1}{4} & 0
\end{array}\right] \\
=\left[\begin{array}{c|cc}
\left(\beta_{1}^{\prime}-\frac{1}{4}\right) \beta_{2}^{\prime}+\left(\beta_{1}^{\prime}+\frac{1}{4}\right) \gamma_{2}^{\prime} & \beta_{1}^{\prime}+\frac{1}{4} & 0 \\
\gamma_{3}^{\prime} & \gamma_{1}^{\prime} & \alpha_{1}^{\prime} \\
\left(\beta_{1}^{\prime}+\frac{1}{4}\right) \beta_{2}^{\prime}+\left(\beta_{1}^{\prime}-\frac{1}{4}\right) \gamma_{2}^{\prime} & \beta_{1}^{\prime}-\frac{1}{4} & 0
\end{array}\right] .
\end{array}
$$

Thus $-\alpha_{1}^{2}=-\alpha_{1}^{\prime 2}$ and so $\alpha_{1}=\alpha_{1}^{\prime}$. Therefore $\psi$ fixes $E_{2}$. Hence $\psi=\rho_{2}(t)$ or $\psi=\varsigma \circ \rho_{2}(t)$ for some $t \in \mathbb{R}$. Now

$$
\begin{array}{r}
\rho_{2}(t) \cdot\left[\begin{array}{c}
\frac{1}{4}+\beta_{1} \\
\gamma_{1} \\
-\frac{1}{4}+\beta_{1}
\end{array}\right]=\left[\begin{array}{c}
\frac{e^{-\theta}}{4}+e^{\theta} \beta_{1} \\
\gamma_{1} \\
-\frac{e^{-\theta}}{4}+e^{\theta} \beta_{1}
\end{array}\right] \\
\left(\varsigma \circ \rho_{2}(t)\right) \cdot\left[\begin{array}{c}
\frac{1}{4}+\beta_{1} \\
\gamma_{1} \\
-\frac{1}{4}+\beta_{1}
\end{array}\right]=\left[\begin{array}{c}
-\frac{e^{-\theta}}{4}-e^{\theta} \beta_{1} \\
\gamma_{1} \\
\frac{e^{-\theta}}{4}-e^{\theta} \beta_{1}
\end{array}\right]
\end{array}
$$

Thus $\gamma_{1}=\gamma_{1}^{\prime}$. Hence $\beta_{1}=\left(\beta_{1}+\frac{1}{4}\right)^{2}-\left(\beta_{1}-\frac{1}{4}\right)^{2}=\left(\beta_{1}^{\prime}+\frac{1}{4}\right)^{2}-\left(\beta_{1}^{\prime}-\frac{1}{4}\right)^{2}=\beta_{1}^{\prime}$. For $\psi=\varsigma \circ \rho_{2}(t)$ we then get $\left(-\frac{e^{-\theta}}{4}-e^{\theta} \beta_{1}\right)+\left(\frac{e^{-\theta}}{4}-e^{\theta} \beta_{1}\right)=-2 e^{\theta} \beta_{1}=2 \beta_{1}$, 
a contradiction. Therefore $\psi=\rho_{2}(t)$. Then $\left(\frac{e^{-\theta}}{4}+e^{\theta} \beta_{1}\right)+\left(-\frac{e^{-\theta}}{4}+e^{\theta} \beta_{1}\right)=$ $2 e^{\theta} \beta_{1}=2 \beta_{1}$. Thus $\theta=0$ and so $\psi$ is the identity automorphism. Therefore $\Sigma_{4, \boldsymbol{\alpha} \boldsymbol{\beta} \boldsymbol{\gamma}}^{(2,1)}$ is equivalent to $\Sigma_{4, \boldsymbol{\alpha}^{\prime} \boldsymbol{\beta}^{\prime} \boldsymbol{\gamma}^{\prime}}^{(2,1)}$ only if $\alpha_{1}=\alpha_{1}^{\prime}, \boldsymbol{\beta}=\boldsymbol{\beta}^{\prime}$, and $\boldsymbol{\gamma}=\boldsymbol{\gamma}^{\prime}$.

Likewise, $\Sigma_{2, \boldsymbol{\beta} \boldsymbol{\gamma}}^{(2,1)}$ is equivalent to $\Sigma_{2, \boldsymbol{\beta}^{\prime} \boldsymbol{\gamma}^{\prime}}^{(2,1)}, \Sigma_{3, \boldsymbol{\beta} \boldsymbol{\gamma}}^{(2,1)}$ is equivalent to $\Sigma_{3, \boldsymbol{\beta}^{\prime} \boldsymbol{\gamma}^{\prime}}^{(2,1)}$, and $\Sigma_{5, \boldsymbol{\alpha} \boldsymbol{\beta} \boldsymbol{\gamma}}^{(2,1)}$ is equivalent to $\Sigma_{5, \boldsymbol{\alpha}^{\prime} \boldsymbol{\beta}^{\prime} \boldsymbol{\gamma}^{\prime}}^{(2,1)}$, respectively, only if $\boldsymbol{\alpha}=\boldsymbol{\alpha}^{\prime}, \boldsymbol{\beta}=\boldsymbol{\beta}^{\prime}$, and $\gamma=\gamma^{\prime}$.

Corollary 4. Every three-input (homogeneous) system is equivalent to exactly one of the following systems

$$
\begin{aligned}
& \Sigma_{1, \boldsymbol{\alpha} \boldsymbol{\beta} \boldsymbol{\gamma}}^{(3,0)}: \gamma_{6} E_{1}+\gamma_{5} E_{2}+\gamma_{4} E_{3}+u_{1}\left(\gamma_{3} E_{1}+\beta_{1} E_{2}+\gamma_{2} E_{3}\right) \\
& +u_{2}\left(\alpha_{2} E_{1}+\gamma_{1} E_{3}\right)+u_{3} \alpha_{1} E_{3} \\
& \Sigma_{2, \boldsymbol{\beta} \boldsymbol{\gamma}}^{(3,0)}: \gamma_{6} E_{1}+\gamma_{5} E_{2}+\gamma_{4} E_{3}+u_{1}\left(\gamma_{3} E_{1}+\beta_{2} E_{2}+\gamma_{2} E_{3}\right) \\
& +u_{2}\left(\left(\gamma_{1}+\beta_{1}\right) E_{1}+\gamma_{1} E_{3}\right)+u_{3}\left(E_{1}+E_{3}\right) \\
& \Sigma_{3, \boldsymbol{\beta} \gamma}^{(3,0)}: \gamma_{6} E_{1}+\gamma_{5} E_{2}+\gamma_{4} E_{3}+u_{1}\left(\gamma_{1} E_{1}+\gamma_{2} E_{2}+\left(\beta_{2}+\gamma_{1}\right) E_{3}\right) \\
& +u_{2}\left(E_{1}+\beta_{1} E_{2}+E_{3}\right)+u_{3}\left(E_{1}+E_{3}\right) \\
& \Sigma_{4, \boldsymbol{\alpha} \boldsymbol{\beta} \boldsymbol{\gamma}}^{(3,0)}: \gamma_{4} E_{1}+\gamma_{4} E_{2}+\gamma_{3} E_{3}+u_{2}\left(\left(\beta_{1}+\frac{1}{4}\right) E_{1}+\gamma_{1} E_{2}+\left(\beta_{1}-\frac{1}{4}\right) E_{3}\right) \\
& +u_{3} \alpha_{1} E_{2}+u_{1}\left(\left(\beta_{2}\left(\beta_{1}-\frac{1}{4}\right)+\gamma_{2}\left(\beta_{1}+\frac{1}{4}\right)\right) E_{1}\right. \\
& \left.+\left(\beta_{2}\left(\beta_{1}+\frac{1}{4}\right)+\gamma_{2}\left(\beta_{1}-\frac{1}{4}\right)\right) E_{3}+\gamma_{3} E_{2}\right) \\
& \Sigma_{5, \boldsymbol{\alpha} \boldsymbol{\beta} \boldsymbol{\gamma}}^{(3,0)}: \gamma_{6} E_{1}+\gamma_{5} E_{2}+\gamma_{4} E_{3}+u_{1}\left(\gamma_{3} E_{1}+\gamma_{2} E_{2}+\left(\beta_{1}+\gamma_{3}\right) E_{3}\right) \\
& +u_{2}\left(E_{1}+\gamma_{1} E_{2}+E_{3}\right)+u_{3} \alpha_{1} E_{2} \text {. }
\end{aligned}
$$

Here $\alpha_{i}>0, \beta_{i} \neq 0$, and $\gamma_{i} \in \mathbb{R}$, with different values of these parameters yielding distinct (non-equivalent) class representatives.

\section{Conclusion}

Two systems (on a connected Lie group G)

$$
\Sigma: \Xi(\mathbf{1}, u) \text { and } \Sigma^{\prime}: \Xi^{\prime}(\mathbf{1}, u)
$$

are detached feedback equivalent (shortly $D F$-equivalent) if there exists a diffeomorphism $\Phi: \mathbf{G} \times \mathbb{R}^{\ell} \rightarrow \mathrm{G} \times \mathbb{R}^{\ell},(g, u) \mapsto(\phi(g), \varphi(u))$ such that

$$
T_{g} \phi \cdot \Xi(g, u)=\Xi^{\prime}(\phi(g), \varphi(u))
$$


for $g \in \mathrm{G}$ and $u \in \mathbb{R}^{\ell}$. It turns out that $\Sigma$ and $\Sigma^{\prime}$ are detached feedback equivalent if and only if there exists a Lie algebra automorphism $\psi \in d$ Aut $(\mathrm{G})$ such that $\psi \cdot \Gamma=\Gamma^{\prime}$ (cf. [10]). Detached feedback equivalence is a weaker equivalence relation than state space equivalence.

A classification, under detached feedback equivalence, of systems evolving on $\mathrm{SO}(2,1)_{0}$ was obtained in [7]. Furthermore a full list of (detached feedback) equivalence representatives was identified. We now compare this classification (under $D F$-equivalence) to the classification obtained in this paper. Specifically, we match (families of) state space equivalence class representatives to detached feedback equivalence class representatives.

For the single-input systems we have

- $\Sigma_{1, \boldsymbol{\alpha} \boldsymbol{\gamma}}^{(1,1)}:\left[\begin{array}{c|c}\alpha_{2} & 0 \\ 0 & 0 \\ \gamma_{1} & \alpha_{1}\end{array}\right]$ is $D F$-equivalent to $\Sigma: \alpha_{2} E_{2}+u E_{3}$;

- $\Sigma_{2, \boldsymbol{\beta} \boldsymbol{\gamma}}^{(1,1)}:\left[\begin{array}{c|c}\beta_{1}+\gamma_{1} & 1 \\ 0 & 0 \\ \gamma_{1} & 1\end{array}\right]$ is $D F$-equivalent to $\Sigma: E_{3}+u\left(E_{2}+E_{3}\right)$

- $\Sigma_{3, \boldsymbol{\alpha} \boldsymbol{\beta} \boldsymbol{\gamma}}^{(1,1)}:\left[\begin{array}{c|c}\beta_{1}+\frac{1}{4} & 0 \\ \gamma_{1} & \alpha_{1} \\ \beta_{1}-\frac{1}{4} & 0\end{array}\right]$ is

- DF-equivalent to $\Sigma: \sqrt{\beta_{1}} E_{1}+u E_{2}$ if $\beta_{1}>0$

- DF-equivalent to $\Sigma: \sqrt{-\beta_{1}} E_{3}+u E_{2}$ if $\beta_{1}<0$.

For the two-input homogeneous systems we have

- $\Sigma_{1, \boldsymbol{\alpha} \boldsymbol{\gamma}}^{(2,0)}:\left[\begin{array}{c|cc}\gamma_{3} & \alpha_{2} & 0 \\ 0 & 0 & 0 \\ \gamma_{2} & \gamma_{1} & \alpha_{1}\end{array}\right]$ and $\Sigma_{1, \boldsymbol{\alpha} \boldsymbol{\gamma}}^{(2,0)}:\left[\begin{array}{c|c}\beta_{1}+\gamma_{1} & 1 \\ 0 & 0 \\ \gamma_{1} & 1\end{array}\right]$ are $D F$-equivalent to $\Sigma: u_{1} E_{2}+u_{2} E_{3}$;

- $\Sigma_{3, \boldsymbol{\alpha} \boldsymbol{\beta} \boldsymbol{\gamma}}^{(2,0)}:\left[\begin{array}{c|cc}\left(\beta_{1}+\frac{1}{4}\right) \gamma_{2} & \beta_{1}+\frac{1}{4} & 0 \\ \gamma_{3} & \gamma_{1} & \alpha_{1} \\ \left(\beta_{1}-\frac{1}{4}\right) \gamma_{2} & \beta_{1}-\frac{1}{4} & 0\end{array}\right]$ is

- DF-equivalent to $\Sigma: u_{1} E_{2}+u_{2} E_{3}$ if $\beta_{1}<0$

- DF-equivalent to $\Sigma: u_{1} E_{1}+u_{2} E_{2}$ if $\beta_{1}>0$. 
For the two-input inhomogeneous systems we have

- $\Sigma_{1, \boldsymbol{\alpha} \boldsymbol{\beta} \gamma}^{(2,1)}:\left[\begin{array}{c|cc}\gamma_{3} & \alpha_{2} & 0 \\ \beta_{1} & 0 & 0 \\ \gamma_{2} & \gamma_{1} & \alpha_{1}\end{array}\right]$ is $D F$-equivalent to $\Sigma:\left|\beta_{1}\right| E_{1}+u_{1} E_{2}+$ $u_{2} E_{3}$

- $\Sigma_{2, \boldsymbol{\beta} \gamma}^{(2,1)}:\left[\begin{array}{c|cc}\gamma_{3} & \beta_{1}+\gamma_{1} & 1 \\ \beta_{2} & 0 & 0 \\ \gamma_{2} & \gamma_{1} & 1\end{array}\right]$ is $D F$-equivalent to $\Sigma:\left|\beta_{2}\right| E_{1}+u_{1} E_{2}+$ $u_{2} E_{3}$

- $\Sigma_{3, \boldsymbol{\beta} \gamma}^{(2,1)}:\left[\begin{array}{c|cc}\gamma_{1} & 1 & 1 \\ \gamma_{2} & \beta_{1} & 0 \\ \beta_{2}+\gamma_{1} & 1 & 1\end{array}\right]$ and $\Sigma_{4, \boldsymbol{\beta} \gamma}^{(2,1)}:\left[\begin{array}{c|cc}\gamma_{3} & 1 & 0 \\ \gamma_{2} & \gamma_{1} & \alpha_{1} \\ \beta_{1}+\gamma_{3} & 1 & 0\end{array}\right]$ are $D F$-equivalent to $\Sigma$ : $E_{3}+u_{1} E_{1}+u_{2}\left(E_{2}+E_{3}\right)$;

- $\Sigma_{5, \boldsymbol{\alpha} \boldsymbol{\beta} \boldsymbol{\gamma}}^{(2,1)}:\left[\begin{array}{c|cc}\left(\beta_{1}-\frac{1}{4}\right) \beta_{2}+\left(\beta_{1}+\frac{1}{4}\right) \gamma_{2} & \beta_{1}+\frac{1}{4} & 0 \\ \gamma_{3} & \gamma_{1} & \alpha_{1} \\ \left(\beta_{1}+\frac{1}{4}\right) \beta_{2}+\left(\beta_{1}-\frac{1}{4}\right) \gamma_{2} & \beta_{1}-\frac{1}{4} & 0\end{array}\right]$ is

- DF-equivalent to $\Sigma: \sqrt{-\beta_{1} \beta_{2}^{2}} E_{1}+u_{1} E_{2}+u_{2} E_{3}$ if $\beta_{1}<0$

- DF-equivalent to $\Sigma: \sqrt{\beta_{1} \beta_{2}^{2}} E_{3}+u_{1} E_{1}+u_{2} E_{2}$ if $\beta_{1}>0$.

The three-input case is trivial; any three-input system is $D F$-equivalent to $\Sigma: u_{1} E_{1}+u_{2} E_{2}+u_{3} E_{3}$.

A summary of the classification results (in matrix form) is appended as a table. 


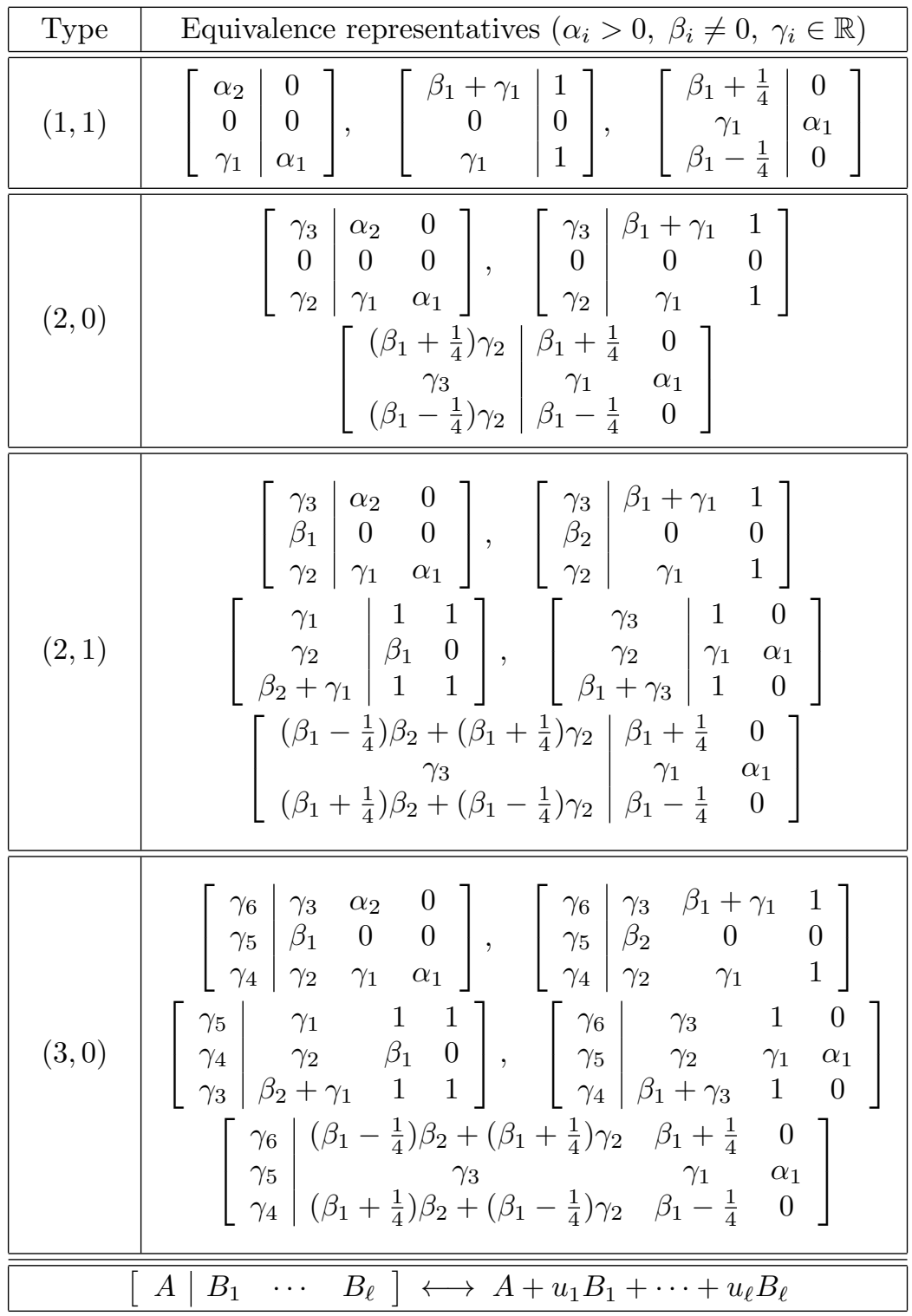

Classification of systems on SO $(2,1)_{0}$ (matrix form) 
Acknowledgement. We would like to acknowledge the involvement of H.C. Henninger in the early stages of this work. The first author is financially supported by the Claude Leon Foundation.

\section{References}

[1] R.M. Adams, R. Biggs and C.C. Remsing, Single-input control systems on the Euclidean group SE (2), Eur. J. Pure Appl. Math. 5(2012), 1-15.

[2] R.M. Adams, R. Biggs and C.C. Remsing, Equivalence of control systems on the Euclidean group SE (2), Control Cybernet. 41(3)(2012), 513-524.

[3] R.M. Adams, R. Biggs and C.C. Remsing, On the equivalence of control systems on the orthogonal group SO (4), in Proc. 8th WSEAS Internat. Conf. on Dynamical Systems \& Control, Porto, Portugal, 2012, pp. 54-59.

[4] R.M. Adams, R. Biggs and C.C. Remsing, Two-input control systems on the Euclidean group SE(2), ESAIM: Control Optim. Calc. Var. 19(4)(2013), 947-975.

[5] A.A. Agrachev and Y.L. Sachkov, Control Theory from the Geometric Viewpoint, Springer, Berlin, 2004.

[6] R. Biggs and C.C. Remsing, A category of control systems, An. Şt. Univ. Ovidius Constanţa 20(1)(2012), 355-368.

[7] R. Biggs and C.C. Remsing, Control affine systems on semisimple three-dimensional Lie groups, An. Ştiinţ. Univ. "A.I. Cuza" Iaşi Mat. 59(2)(2013), 399-414.

[8] R. Biggs and C.C. Remsing, Control affine systems on solvable threedimensional Lie groups, I, Arch. Math. (Brno) 49(3)(2013), 187-197.

[9] R. Biggs and C.C. Remsing, Control affine systems on solvable threedimensional Lie groups, II, Note Mat. 33(2)(2013), 19-31.

[10] R. Biggs and C.C. Remsing, On the equivalence of control systems on Lie groups, Commun. Math. 23(2)(2015), 119-129.

[11] R. Biggs and C.C. Remsing, On the equivalence of cost-extended control systems on Lie groups, in Proc. 8th WSEAS Internat. Conf. on Dynamical Systems 83 Control, Porto, Portugal, 2012, pp. 60-65.

[12] R.W. Brockett, System theory on group manifolds and coset spaces, SIAM J. Control 10(2)(1972), 265-284. 
[13] I. Caşu, M. Craioveanu, M. Puta and A. Sima, The rigid body on $\operatorname{SO}(2,1)$ and its integration, Tensor (N.S.) 68(2007), 76-81.

[14] V.V. Gorbatsevich, A.L. Onishchik and E.B. Vinberg, Foundations of Lie Theory and Lie Transformation Groups, Springer, Berlin, 1997.

[15] B. Jakubczyk, Equivalence and Invariants of Nonlinear Control Systems, in Nonlinear Controllability and Optimal Control (Sussmann H.J, ed.), M. Dekker, New York, 1990, pp. 177-218.

[16] V. Jurdjevic, Geometric Control Theory, Cambridge University Press, Cambridge, 1997.

[17] V. Jurdjevic and H.J. Sussmann, Control systems on Lie groups, J. Diff. Equations 12(1973), 313-329.

[18] A.L. Onishchik and E.B. Vinberg, Lie Groups and Lie Algebras III, Springer, Berlin, 1994.

[19] M. Puta and A. Sima, An optimal control problem on the Lie group SO(2,1), An. Univ. Vest Timiş. Ser. Mat.-Inform. 44(2006), 109-117.

[20] M. Puta, C. Hedrea and A. Sima, Controllability problems in the rigid body dynamics on $\mathrm{SO}(2,1)$, An. Univ. Vest Timiş. Ser. Mat.-Inform. 44(2006), 127-134.

[21] M. Puta and A. Sima, The rigid body dynamics on $\operatorname{SO}(2,1)$, Tensor (N.S.) 67(2006), 182-189.

[22] C.C. Remsing, Optimal control and Hamilton-Poisson formalism, Int. J. Pure Appl. Math. 59(2010), 11-17.

[23] W. Respondek and I.A. Tall, Feedback Equivalence of Nonlinear Control Systems: a Survey on Formal Approach, in Chaos in Automatic Control, CRC Press, Boca Raton, FL, 2006, pp. 137-262.

[24] Y.L. Sachkov, Control theory on Lie groups, J. Math. Sci. 153(2009), $381-439$.

Rory BIGGS,

Department of Mathematics,

Rhodes University,

PO Box 94, 6140 Grahamstown, South Africa.

Email: rorybiggs@gmail.com

Claudiu C. REMSING,

Department of Mathematics,

Rhodes University,

PO Box 94, 6140 Grahamstown, South Africa.

Email: c.c.remsing@ru.ac.za 